\title{
A Novel Digital Watermarking Technique for Video Copyright Protection
}

\author{
Poulami Ghosh ${ }^{1}$, Rilok Ghosh ${ }^{2}$,Souptik Sinha ${ }^{3}$, Ujan Mukhopadhyay ${ }^{4}$, \\ Dipak kr. Kole ${ }^{5}$ and Aruna Chakroborty ${ }^{6}$ \\ Department of Computer Science \& Engineering \\ St. Thomas' College of Engineering and Technology \\ Kolkata-700023,India \\ ${ }^{1}$ poulami.ghosh90@gmail.com, ${ }^{2}$ rlkghshegmail. $\mathrm{com}^{3}$ \\ ${ }^{3}$ souptiksinhaonly1.001@gmail.com, ${ }^{4}$ ujan.kush@gmail.com, \\ 5 dipak.kole@gmail.com, ${ }^{6}$ aruna.stcet@gmail.com
}

\begin{abstract}
:
Due to the rapid growth of internet and technology, protecting digital data is becoming very urgent. In this paper a novel watermarking technique is proposed where both visible and invisible watermarks are embedded in a video. Digital data can be copied easily without any degradation in quality, so the protection of the data is necessary. Digital watermarking is a technology to embed additional information into the host signal to ensure security and protection of multimedia data. The video frames contain both the watermarks, so it is more robust to attacks. The watermarking scheme described here deals with embedding and extraction of the watermarks. Discrete Wavelet transform (DWT) is used to embed the invisible watermark and Peak Signal to Noise Ratio (PSNR) is calculated to measure efficiency of this method.
\end{abstract}

Key Terms: Digital Watermarking, Visible Watermarking technique and Discrete Wavelet Transform

\section{Introduction}

In the past few years, digital multimedia distribution over the Internet has grown rapidly as a result of the latest developments in technologies. Due to the continuously increasing availability of the Internet, the digital data can be easily shared, processed or used causing serious security problems. As a solution to this problem, different authentication techniques are used.

Digital Right Management (DRM) systems are used that help the owners to protect their data and restrict their distributions. Digital watermarking techniques are used to add some data (watermark) into the original signal in order to protect it.

Digital watermarking is the process of adding information into multimedia data also called original media. The digital watermarking techniques are used to protect the digital data from

Natarajan Meghanathan, et al. (Eds): SIPM, FCST, ITCA, WSE, ACSIT, CS \& IT 06, pp. 601-609, 2012.

(C) CS \& IT-CSCP 2012

DOI : $10.5121 /$ csit.2012.2360 
illegal copying by embedding some information into the multimedia. A watermark is embedded into the host signal (e.g. - image, video, audio) that can be extracted later to verify identity of the owner. Digital video watermarking can be of two types - visible watermarking and invisible watermarking [1]. In case of visible watermarking, the logo or the information appears in the video frames being completely visible or partially visible. In invisible watermarking, the information should not appear in the video i.e., it must be perceptually invisible [2]. The invisible watermarking provides more security to video, though the visible watermarks protect the digital data in more active manner.

Watermarking techniques can be classified as- Spatial domain and Frequency domain Watermarking Techniques. Spatial domain techniques can be Correlation based where different correlation properties are used such as adding noise, or adding pixels and Least Significant Bit (LSB) modification techniques where the lowest order bit of selected pixels are changed. Frequency domain techniques, also called transform domain are more robust than spatial domain techniques and can be of three types DWT (Discrete Wavelet Transform), DCT (Discrete Cosine Transform), DFT (Discrete Fourier Transform). DWT is more efficient than the other transform domain techniques [3].

In this paper we will focus on a technique that will protect a video from illegal access by embedding both visible and invisible watermarks into it. An invisible watermark protects a video from being copied and manipulated from the internet, but if someone takes a copy of that video using a camera then he will be able to claim it as his own video. To protect the video from this type of attacks, a visible watermark will be incorporated in the video which will appear in the video frames randomly depending upon the system and will be partially visible. DWT is used to embed the watermark in the video frames imperceptibly. The visible watermark is embedded depending on the user's choice or randomly. As the video contain both invisible and visible watermarks so this algorithm will provide more security to the owner's video.

This paper has the following sections: Section II contains brief descriptions about DWT and Visible Watermarking techniques, Section III deals with the block diagrams and descriptions of the embedding and extraction procedures, Section IV has the experimental results and lastly, Section V gives the conclusion.

\section{Watermarking Schemes}

\section{A. Visible Watermarking}

Visible watermark appears in the video frames either continuously or randomly. It should be partially visible in the video frames, so that the quality of the video is not degraded and also should be difficult and perhaps impossible to remove from the video frames. It protects the video in a different manner than that of the invisible watermarking techniques. If someone captures the video using a camera, then the visible watermarks should randomly appear on the frames which the attacker cannot remove easily.

\section{B. Discrete Wavelet Transform}

The most advanced and useful transform domain watermarking technique is Discrete Wavelet Transform (DWT). DWT is a hierarchical transform. DWT offers multi resolution analysis i.e., it has the capabilities to study or analyze a signal at different levels. Wavelets are localized waves; 
they are generated from a mother wavelet through translation and scaling. The wavelets produced from a mother wavelet $\psi(\mathrm{t})$, are denoted by,

$$
\Psi_{\mathrm{a}, \mathrm{b}}(t)=\frac{1}{\sqrt{a}} \Psi\left(\frac{t-b}{a}\right),
$$

where $\mathrm{a}$ and $\mathrm{b}$ are two arbitrary real numbers denoting the parameters for scaling and translation in the time axis respectively.

We can divide a signal into a set of wavelets by using DWT. Two functions are required which are Scaling function (filters the lowest level of transform), denoted by $\varphi(\mathrm{x})$ and Wavelet function (scales each level half its bandwidth), denoted by $\psi(\mathrm{x})$. 1D-DWT can separate the high and the low-frequency portions of a signal by using filters. 2D-DWT divides a signal into a set of four components. These are LL (the lower resolution approximation component), HL (horizontal), LH (vertical) and $\mathrm{HH}$ (diagonal) detailed component. We will use the LL and HH components in our algorithm to embed watermark in video frames. DWT is more computationally efficient than other transform methods like DFT and DCT for its excellent spatial-frequency localization properties $[4,5,6,7,9]$.

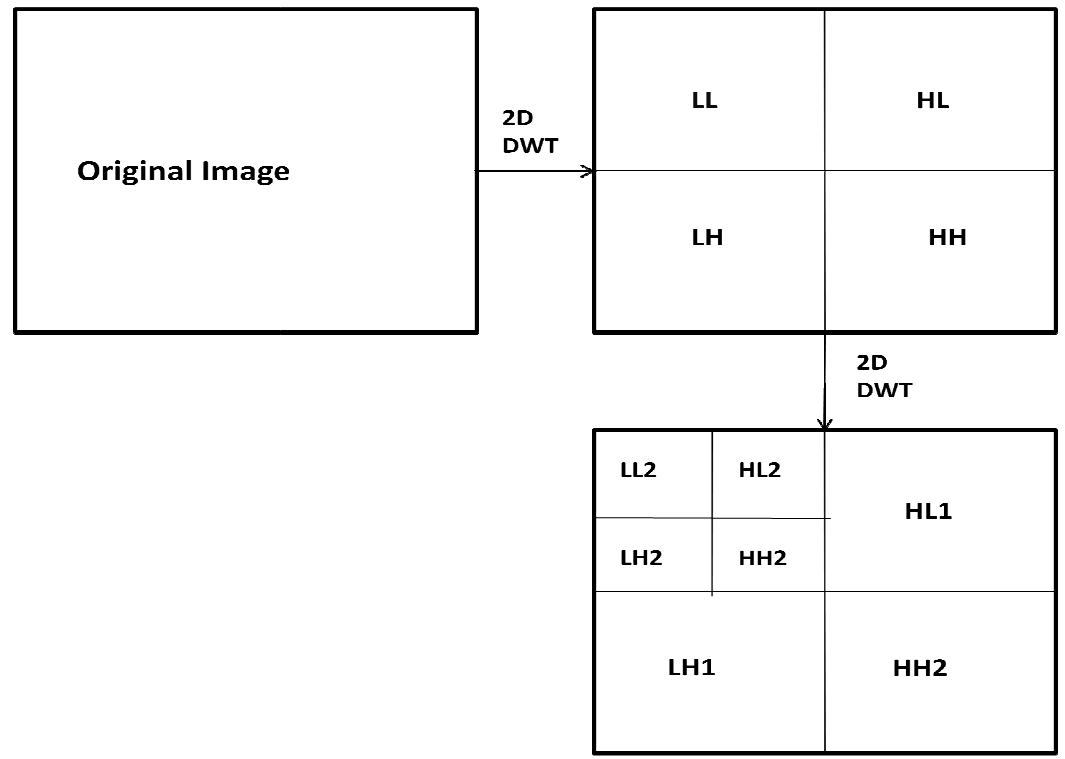

Fig 1- A two scale DWT

\section{Proposed Technique}

The method described in this paper can be divided into two parts - embedding and extraction. The invisible watermark is embedded in the video using DWT. Due to its excellent timefrequency analysis properties, DWT is used to select the regions where the watermark can be embedded invisibly. To each of the video frames DWT is applied. Then the watermark is embedded in the transformed coefficients of the video frames. For the visible watermark, the user can either give the choice of the region for embedding the watermark (for e.g. - top, bottom or middle) or the watermark can appear randomly. After the user gives the input, the watermark will be embedded in the frames being partially visible. A compound mapping will be used for visible 
watermarking [8]. Extraction will be done for both the invisible and visible watermarks. Then the PSNR will be calculated to prove the efficiency of this method.

\section{Algorithms-}

The invisible and the visible watermarks are embedded using the following procedure.

Embedding the invisible watermark:

1. Take the uncompressed video as input.

2. Read each frame of the video.

3. Apply 2D-DWT on the frames.

4. Again apply 2D-DWT on the HH component of the video frames.

5. Embed the watermark.

6. Apply inverse 2D DWT respectively.

Embedding the visible watermark:

1. Take the invisible watermarked video as input for embedding visible watermark

2. Select watermark logo.

3. Read the number of frames in video

4. Select the single frame from the input video

5. Take the user input to select the visible watermarking area.

6. Read the pixel of watermarking area $x$ of video frame.

7. Read the next pixel of the watermarking area y of video frame.

8. Determine the difference $\mathrm{z}$.

9. Read the pixel value of watermark $t$.

10. Replace the watermark pixel by adding $(\mathrm{z}+\mathrm{t})$

12. Embed the watermark in alternative video frame.

13. Combine the video frames

14. Play the visible watermarked video. 
Fig. 2 shows the block diagram of the proposed method.

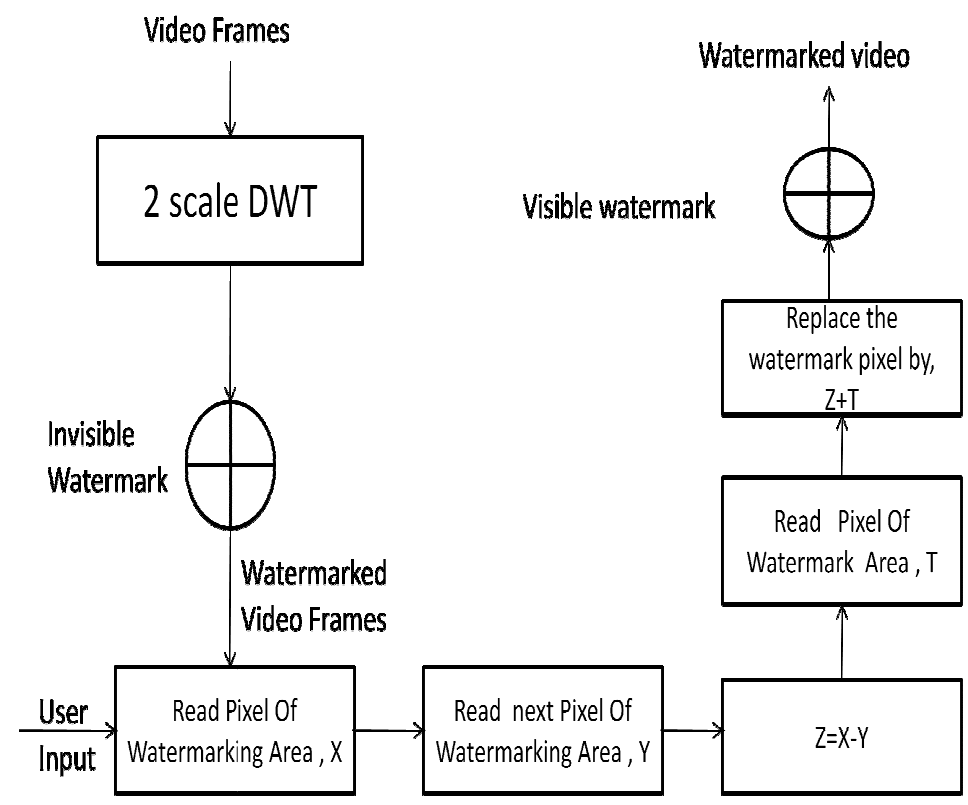

Fig. 2- Flow chart of the above procedure

The extraction of the invisible watermark is done by the following method.

Extracting the invisible watermark:

1. Take the original and embedded video as input.

2. Take the video frames.

3. Apply 2-D dwt on the video frames.

4. Again apply 2-D dwt on the HH component of the resultant video frames

5. Subtract original video frame from the embedded video frame. 
Fig. 3 shows the flowchart for the extraction procedure

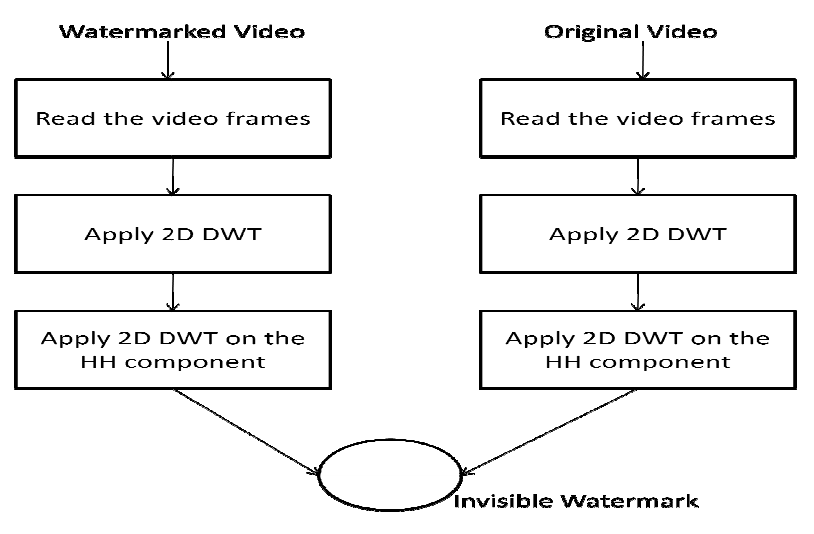

Fig 3-Flowchart for the extracting process

\section{Experimental Results}

This invisible and visible watermarking technique is applied on a gray level video. Fig 4 and Fig 5 is the watermark logo before embedding in a video frame and after extracting from that video frame respectively. Fig 6, Fig 7, Fig 8 denotes the video frames after embedding the visible watermark at different positions, depending on the user. The method is user friendly as the user can choose positions in the frame to embed the watermark. The video frames contain both invisible and visible watermarks, so the video is more robust to attacks.

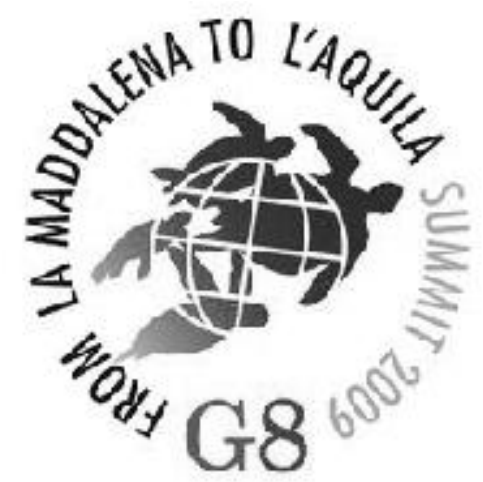

Fig. 4 - Original Watermark 


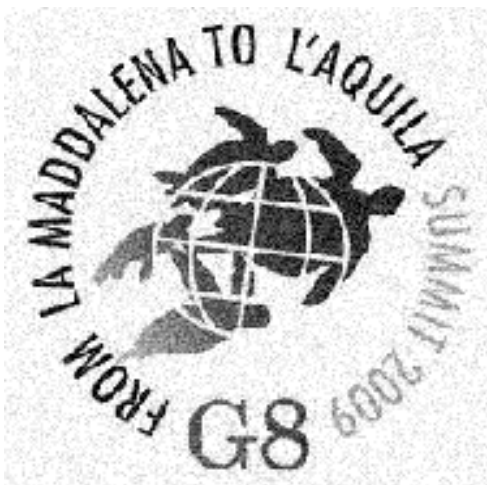

Fig. 5 - Extracted Watermark

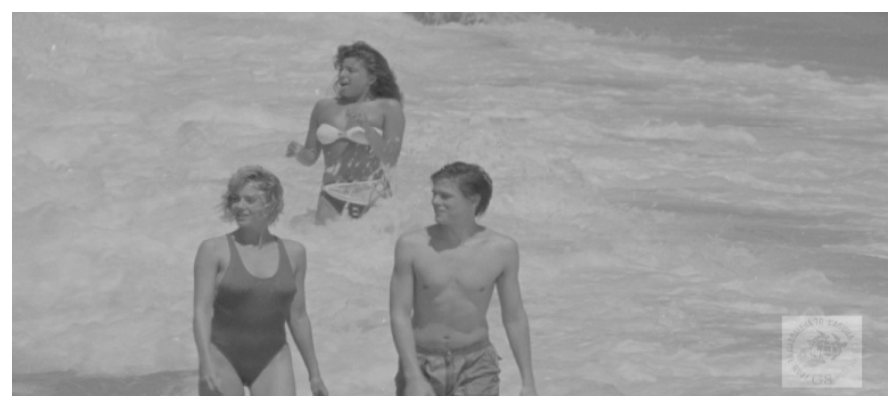

Fig. 6- Watermark at bottom

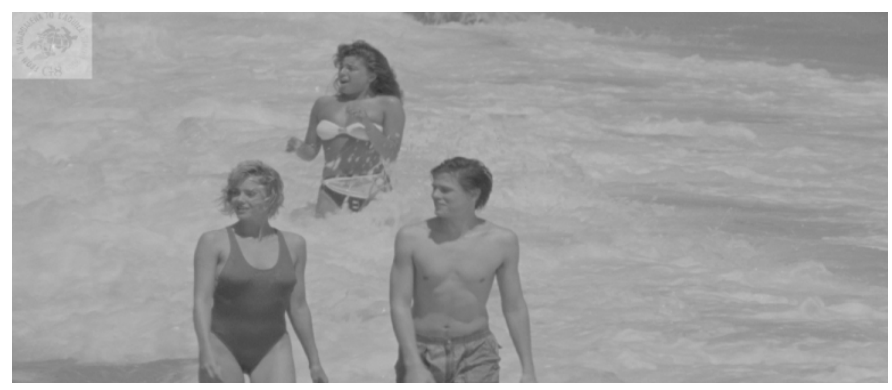

Fig. 7- Watermark at top

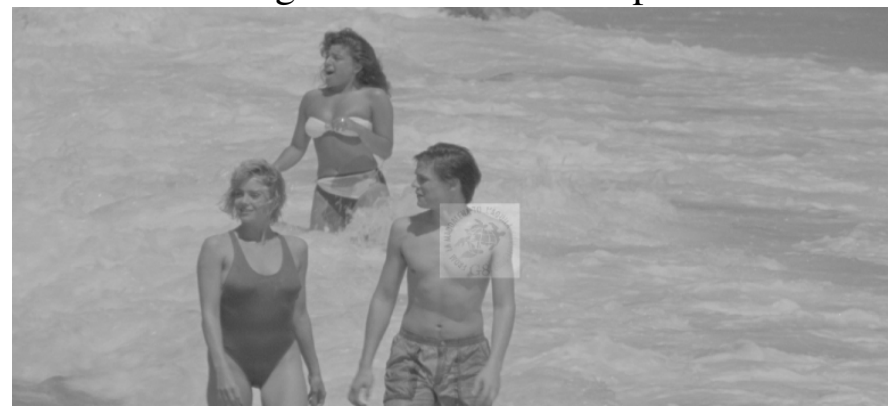

Fig. 8- Watermark at middle 
The robustness of the video frames is measured in terms of different attacks on the video frames like 'salt \& pepper noise', 'Gaussian noise', 'median filtering' and 'contrast adjustment'[10]. PSNR is calculated after the different attacks are applied.

Table1 shows the PSNR values of the video frames after the attacks.

Table1

\begin{tabular}{|l|l|}
\hline Attacks & PSNR \\
\hline Salt \& pepper noise & 28.754 \\
\hline Gaussian noise & 28.75 \\
\hline Contrast adjustment & 34.39 \\
\hline Median filtering & 34.36 \\
\hline
\end{tabular}

\section{Related Works}

The PSNR values obtained after applying different attacks makes it evident that proposed watermarking algorithm is robust again these attacks. This algorithm is done in Matlab platform, work can be done to implement this method in other platforms as well (like C, OpenCV etc.). In future, some work can be done using this algorithm other data such as various texts and images. This type of data can also be protected from copying by embedding watermarks according to the algorithm described in this paper.

\section{Conclusion}

In this technique we are including both visible and invisible watermark which gives an extra edge in the copyright protection. As we are using compound mapping to embed the visible watermark it helps to increase the robustness of the video. The proposed algorithm works well on gray scale and on video of uncompressed avi format. In future work will be done on the colored video, so that the approached method works well on all types of videos. 


\section{References}

[1] Dr.M.A.Dorairangaswamy, "A Novel Invisible and Blind Watermarking Scheme For Copyright Protection of Digital Images", International Journal of Computer Science and Network Security, VOL.9 No.4, April 2009.

[2] Dr.M.Mohamed Sathik, S.S.Sujatha, "An Improved Invisible Watermarking Technique for Image Authentication", International Journal of Advanced Science and Technology Vol. 24, November, 2010.

[3] Shivani Khurana, "Watermarking and Information-Hiding", International Journal of Computer Science and Information Technologies, Vol. 2 (4) ,1679-1681, 2011.

[4] Mahmoud El-Gayyar and Prof. Dr. Joachim von zur Gathen, "Watermarking Techniques Spatial Domain Digital Rights Seminar", Media Informatics, University of Bonn, Germany, May 06.

[5] S. Sinha, S. Pramanick, A. Jagatramka, P. Bardhan, D.K. Kole, A.Chakraborty, "Digital Video Watermarking using Discrete Wavelet Transform and Principal Component Analysis", International Journal of Wisdom Based Computing, Vol. 1 (2), 7-12, August 2011.

[6] C.H. Li and S.S. Wang, "Transform-Based Watermarking for Digital Images and Video," IEEE, International Conference on Consumer Electronics, June1999.

[7] Elham Shahinfard, Shohreh Kasaei, "Digital Image Watermarking using Wavelet Transform".

[8] Saraswathi.M, "Lossless Visible Watermarking for Video", International Journal of Computer Science and Information Technologies, Vol. 2 (3) ,1109-1113, 2011.

[9] H. Liu, N. Chen, J. Huang, “A Robust DWT-Based Video Watermarking Algorithm”, Proceedings of the International Conference on Signal and Image Processing Applications (ICSIPA), IEEE, pp. 352 356,2000 .

[10] T.JAYAMALAR, Dr. V. RADHA, "Survey on Digital Video Watermarking Techniques and Attacks on Watermarks", International Journal of Engineering Science and Technology, Vol. 2(12), 69636967, 2010. 